\title{
INDICATIONS ET RESULTATS DE LA SEPTOPLASTIE CHEZ L'ENFANT
}

\author{
K. MIGHRI, N. B. HAMIDA, CH. EL AOUD, H. CHRAITI*, I. LAHMAR, N. DRISS. \\ SERVICE ORL CHU MAHDIA. TUNISIE \\ *UNITÉ D'ANESTHÉSIE RÉANIMATION CHU MAHDIA. TUNISIE
}

\begin{abstract}
La septoplastie chez l'enfant est un sujet de controverse jusqu'à nos jours.
Notre étude est rétrospective à propos de 47 cas colligés au service ORL de l'hôpital Tahar Sfar de Mahdia sur une période de 19 ans (1988-2006). L'âge moyen de nos malades est de 13,8 ans. Le sexe masculin est prédominant (63,8\%). Tous nos malades ont bénéficié d'un examen rhinologique et un bilan radiologique standard. A côté de la déviation de la cloison nasale nous avons noté une déviation de la pyramide nasale chez 4 malades. Tous nos malades ont bénéficié d'une septoplastie sous anesthésie générale. Après un recul moyen de 2 ans nous avons un bon résultat fonctionnel (fosses nasales perméables) chez 37 malades.
\end{abstract}

Mots-clés : Obstruction nasale, septoplastie, enfant.

Septoplasty in children is a subject of many discussions.

We tray to identify the main indication of septoplasty in children.

Our study is a retrospective study of 47 cases. The indications should be limited to severe nose obstructions with major septal deviation.

Keywords : Nasal destruction, septoplasty, children.

\section{INTRODUCTION}

Opérer un nez en pleine croissance reste un problème difficile à aborder du fait du retentissement possible sur le développement cranio-facial de l'enfant.

Certains auteurs optent pour une chirurgie septale à minima. A travers une étude de 47 cas on se propose de dégager les indications de la septoplastie chez l'enfant et de préciser l'évolution

\section{MATÉRIEL ET MÉTHODES}

Notre étude est rétrospective à propos de 47 cas colligés au service ORL de l'hôpital Tahar Sfar de Mahdia sur une période de 19 ans (1988-2006).

Pendant cette période nous avons réalisé 396 septoplasties ce qui représente un taux de $11,8 \%$.

Nous avons étudié les dossiers et les comptes - rendus opératoires des malades.

\section{RESULTATS}

L'âge moyen de nos malades a été de 13,8ans avec des extrêmes allant de 8 à 16 ans.

Trente sept de nos malades étaient de sexe masculin. Le traumatisme nasal direct a été retrouvé dans $57,5 \%$. La symptomatologie fonctionnelle a été dominée par l'obstruction nasale retrouvée chez 43 malades. Elle a été bilatérale chez 13 malades droites chez 20 malades et gauche chez 14 malades. L'obstruction nasale a été totale chez 30 malades et partielle chez 17 malades.

Chez 4 malades la déformation de la pyramide nasale a été le motif de consultation.

Les signes d'hyper réactivité nasale à type de prurit nasal ont été retrouvés chez 6 malades.
A l'examen physique nous avons trouvé une déviation de la cloison nasale en verre de montre chez 20 malades, en coup de hache chez 13 malades et en s'étalique chez 13 malades.

Chez 4 malades une déviation aussi bien de la cloison que de la pyramide nasale ont été retrouvé.

Les déformations cranio-faciales associées à type de fente vélo-palatine ou de défaut de fermeture des cissures n'ont pas été mentionnées.

Une pathologie otologique a été trouvée chez 15 malades. II s'agissait d'une otite séro-muqueuse chez 10 malades et une otite moyenne chronique chez 5 malades. Tous nos malades ont bénéficié d'une septoplastie sous anesthésie générale.

II s'agissait d'une résection cartilagineuse à minima (sans toucher à l'os).

Chez 4 malades nous avons réalisé en plus une rhinoplastie (réduction d'une bosse) en utilisant la rappe.

Après un recul moyen de 2 ans nous avons noté un bon résultat fonctionnel chez 37 malades soit $78,7 \%$ en effet les malades n' ont pas de gène fonctionnelle et à l'examen rhinologique les fosses nasales sont perméables Quatre malades ont nécessité une reprise chirurgicale devant la persistance de l'obstruction nasale un an après la première intervention soit à l'âge de 15 ans.

Six malades ont été perdus de vue.

\section{DISCUSSION}

La septoplastie est une geste facile qui s'adresse à corriger une déviation de la cloison nasale responsable d'une obstruction nasale. (1)

Sa pratique chez l'enfant doit être prudente vu le risque de perturber la croissance cranio-faciale. 
Cette septoplastie doit être bien réfléchie. (2)

La déformation septale est le plus souvent secondaire à un traumatisme, rarement à une malformation congénitale.

L'obstruction nasale chez l'enfant a des répercussions aussi bien sur le développent cranio-facial que l'appareil respiratoire avec parfois des troubles du sommeil allant jusqu'au syndrome d'apnée du sommeil. (2, 3, 4)

Un tel syndrome a des répercussions sur l'activité scolaire de l'enfant. En effet le syndrome d'apnée de sommeil est à l'origine des micro-éveils nocturne perturbant ainsi la sécrétion de l'hormone de croissance.

La septoplastie chez l'enfant doit obéir à certaines règles (5): - la chirurgie doit être extra muqueuse il ne faut pas toucher à la muqueuse risque de synéchies.

- le respect de la partie antérieure de la cloison (columelle).

- le respect de la continuité du septum surtout en antéroinférieur et les zones de contact avec la lame perpendiculaire de l'ethmoïde et du vomer.

En respectent ces règles les indications de la septoplastie chez l'enfant sont de deux ordres : $(1,5)$

* les déviations sévères de la cloison nasale post-traumatique ou malformative.

* la crainte d'un défaut de développement cranio-facial.

La pratique des ostéotomies est déversement appréciée par les auteurs ; pour certains les ostéotomies sont moins dangereuses que la septoplastie pour l'enfant.

D'autres points restent à discuter tels que le terrain : fautil opérer la cloison d'une enfant allergique ? A partir de quel âge faut-il opérer ?

La majorité des auteurs sont d'accord pour une bonne préparation préopératoire d'un enfant allergique (anti-histaminiques, désensibilisation) (4).

L'âge de 7-8 ans parait raisonnable pour intervenir sur la cloison nasale (1).
L'évolution est le plus souvent favorable à part quelques complications classiques telles que l'hématome ou l'infection.

La perforation septale et les synéchies doivent être prises en charge rapidement.

L'évolution peut être évaluée de façon subjective par la vérification de la perméabilité nasale ou objective par une rhinomanométrie.

Dans notre série le taux d'échec est de $21,3 \%$ il s agissait dune reprise chirurgicale chez quatre malades et des synéchies chez six malades nécessitant une libération sous anesthésie générale (tableau I).

\begin{tabular}{|c|c|c|c|c|}
\hline Auteurs & Nombres & Recul (an) & $\begin{array}{c}\text { Bon résultut } \\
\%\end{array}$ & $\begin{array}{c}\text { Echec } \\
\%\end{array}$ \\
\hline Crysdal (6) & 15 & 5 & 67 & 25 \\
\hline Slobodan(7) & 24 & 5 & 83 & 17 \\
\hline Ch. M'barek(5) & 40 & 2 & 85 & 15 \\
\hline Notre série & 47 & 2 & 78,7 & 21,3 \\
\hline
\end{tabular}

Tableau I: Résultats fonctionnels des septoplasties de l'enfant.

\section{CONCLUSION}

La septoplastie chez l'enfant doit être soigneusement discutée.

L'obstruction nasale sévère et la crainte d'une déformation cranio-faciale sont les principales indications.

Techniquement il faut être le plus conservateur possible. L'évolution peut être émaillée de complications telles que la perforation septale et les synéchies.

\section{REFERENCES}

1- Levaillez J.. L'obstruction nasale néonatale. Arch. Pediatr 2001; 8:214-20 2 - El Hakim H. , Crysdale W., Abdallah M et al.

A study of anthropometric measures before an after external septoplasty in children. Archives of otolaryngology head and neck surgery 2001; 127: 11 3 - A. J. Emami, Linda Bradsky, Michael Pizzuto

Neonatal septoplasty : case report and review of the literature.

International journal of Pediatric Otorhinolaryngology 1996, 35, 271-275

4-J. Percodani, A Didier, E.Serrano. L'obstruction nasale de l'enfant asthmatique:

du diagnostic à la prise en charge. Revue française d'allergologie et d'immuno- logie clinique 2004 ; 44, 590-596.

5- M'barek Ch.; Hrigua I. , kharrat S. et al. Les septoplasties de l'enfant J. Tun ORL et chir cervico-faciale $2008 ; 8: 21-24$

6- Crysdale W. Externel septo-rhinoplasty in children. Laryngoscope . 1985;95:12-6.

7- Slobodan B., Jugo M.D.Total septal reconstruction through decortication (external) approach in children. Arch. oto laryngeal head neck surg 1987 ; 90:154-158 